\title{
Use of Botulinum Toxin for Androgenic Alopecia: A Systematic Review
}

\author{
Robert S. English Jr. Sophia Ruiz \\ Perfect Hair Health, San Francisco, CA, USA
}

\section{Keywords}

Botulinum toxin · Androgenic alopecia · Hair loss

\begin{abstract}
In this systematic review, we summarize the efficacy and safety of intradermal and intramuscular botulinum toxin injections for androgenic alopecia (AGA). Using PubMed, we conducted a literature search up to February 2021 using the following keyword combinations: "botulinum toxin" or "botox" and "androgenetic alopecia," "hair loss," or "alopecia." Five clinical studies met our inclusion criteria: 4 prospective cohorts and 1 randomized clinical trial (RCT). Study durations ranged from 24 to 60 weeks. No studies included control groups or compared botulinum toxin injections against approved treatments. A total of 165 participants were identified - all of whom were males with AGA. Of the 4 studies measuring response rates (i.e., subjects with $>0 \%$ hair changes), response rates ranged from 75 to $79.1 \%$. Within studies measuring hair count changes from intramuscular injections, changes ranged from 18 to $20.9 \%$. No serious adverse events were reported. Studies on botulinum toxin injections have produced favorable outcomes for AGA subjects. However, results should be interpreted with caution due to the absence of control groups, small numbers of participants, and relatively low Jadad quality scores. Large RCTs are recommended to confirm efficacy and safety, explore the ef-
\end{abstract}

(C) 2021 The Author(s)

Published by S. Karger AG, Basel

This article is licensed under the Creative Commons Attribution 4.0 International License (CC BY) (http://www.karger.com/Services/ OpenAccessLicense). Usage, derivative works and distribution are permitted provided that proper credit is given to the author and the original publisher. fects of botulinum toxin on females with pattern hair loss, and establish best practices for intradermal and intramuscular injection methodologies.

(C) 2021 The Author(s).

Published by S. Karger AG, Basel

\section{Introduction}

Androgenic alopecia (AGA) is a common hair loss disorder that predominantly affects regions above the galea aponeurotica. Consensus is that AGA is mediated by genetics and androgens [1]. In particular, the action of dihydrotestosterone (DHT) on genetically sensitive hair follicles appears to dysregulate the Wnt/beta-catenin pathway, induce transforming growth factor beta 1 (TGF- $\beta 1$ ), and trigger apoptosis in dermal papillae cells (DPCs) - leading to a shortened hair cycle, an increased telogen:anagen ratio, and the progressive miniaturization of hair follicles following each re-entry into anagen [2-4].

Two approved treatments for AGA are oral finasteride and topical minoxidil. Finasteride and minoxidil reduce $5 a-D H T$ and improve vasodilation, respectively [1]. Clinical studies demonstrate that $1 \mathrm{mg}$ daily of finasteride slows, stops, or partially reverses AGA progression in 80 $90 \%$ of men and, over a 2-year period, improves hair counts by $10 \%[5,6]$. In photographic assessments, twice daily applications of $5 \%$ minoxidil have demonstrated 
hair regrowth in $38.4 \%$ of participants [7]. However, finasteride remains mainly limited to stopping AGA progression [6]. Moreover, minoxidil efficacy appears to wane over time [8]. As such, there is demand for novel and more effective AGA treatments.

While evidence strongly implicates DHT in the pathogenesis of AGA, researchers continue to explore additional biological, histological, and morphological factors that may contribute to or accelerate the balding process. One such area of interest involves the scalp perimeters muscles anchored to the galea aponeurotica - the occipitalis, frontalis, auricularis, and temporalis - of which investigators often report are chronically and involuntarily contracted in AGA-affected males. The temporalis and auricularis intersect with supraorbital and supratrochlear arterial branches. When chronically contracted, these muscles may pinch vascular networks that indirectly supply blood and oxygen to AGA-prone tissues [9]. Moreover, two-dimensional von Mises models suggest that when the occipitalis and frontalis are contracted, the tensile patterns generated across the galea aponeurotica closely align with the patterning and progression of male AGA [10].

Reductions to blood flow and transcutaneous oxygen levels have been observed in AGA tissues [9, 11]. Moreover, increased activity of androgens, androgen-mediated TGF- $\beta 1$, and/or fibrosis are observed not only in AGAaffected sites, but also in tissues under chronic inflammation, contraction, or pressure: the eyelids of patients with Graves' disease, the prostate of men with benign prostate hyperplasia, tendons affected by Dupuytren's contracture, and diseased periodontal tissues [12-15]. As such, there is therapeutic interest in targeting to reduce the contraction of scalp perimeter muscles in AGA subjects. One such intervention involves intradermal and/or intramuscular injections with botulinum toxin.

Botulinum toxins are protein neurotoxins produced by Clostridium bacteria. Botulinum toxins trigger dosedependent respiratory and musculoskeletal paralysis by inhibition of neurotransmitter action [16]. Initially, these neurotoxic effects were noted for their involvement in flaccid paralysis of botulism. Over the last 3 decades, investigators have demonstrated the therapeutic value of botulinum toxin - especially serotype A - for selective paralysis of muscles involved in a variety of disease states and cosmetic concerns. In particular, injections of botulinum toxin type A have demonstrated clinical efficacy for the treatment of facial wrinkles, hyperhidrosis, spasticity management, temporomandibular disorders, migraines, and excessive salivary secretion [16, 17]. More

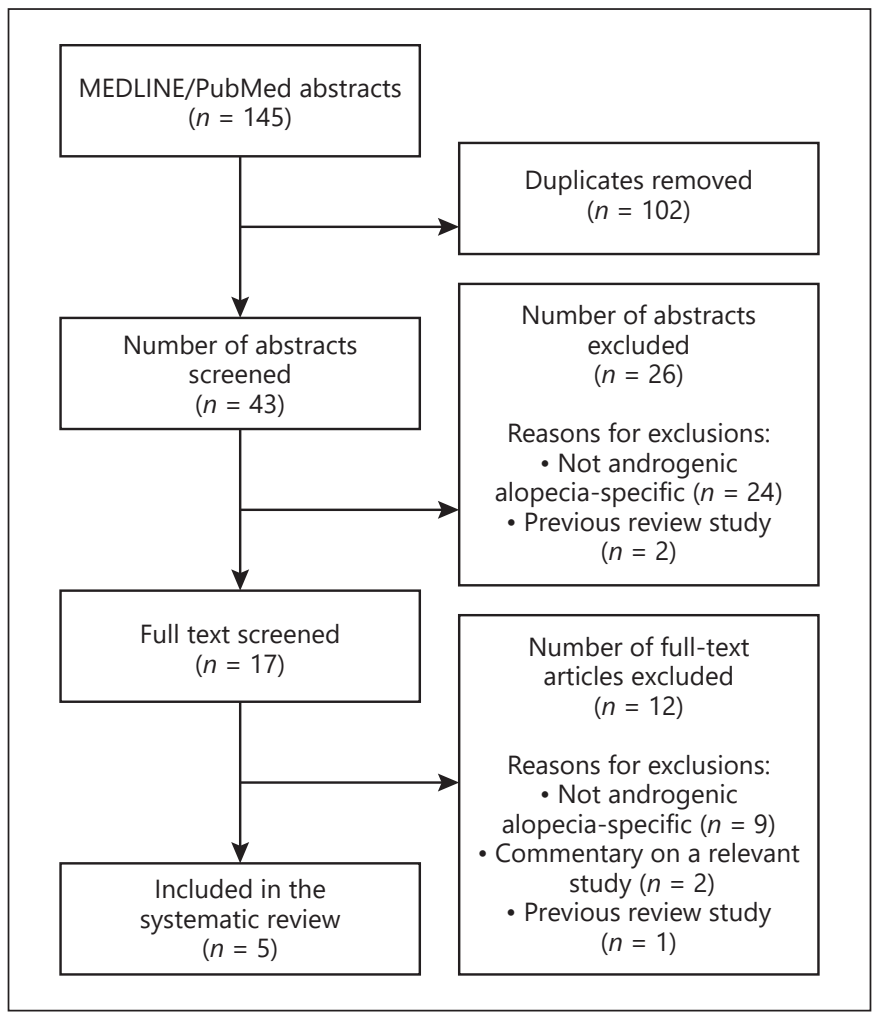

Fig. 1. A PRISMA flowchart detailing the identification, screening, eligibility, and inclusion decisions for our systematic review on botulinum toxin for the treatment of androgenic alopecia.

recently, investigators have begun conducting clinical trials on botulinum toxin as an intervention for AGA.

In this systematic review, we explore the available clinical studies on botulinum toxin for the treatment of AGA. We evaluate study designs, methodologies, and outcomes - as well as evidence quality using Jadad scoring. Finally, we discuss possible mechanisms in which intramuscular and/or intradermal botulinum toxin may improve AGA outcomes, identify limitations in the current body of research, and provide recommendations for future clinical trials.

\section{Methods}

\section{Literature Search}

A literature search was conducted using PubMed up to February 2021 to identify original articles evaluating the effects of intramuscular botulinum toxin injections on AGA. The database searches used combinations of the following keywords: "botulinum toxin" or "botox" and "androgenetic alopecia," "hair loss," or "alopecia."
English Jr./Ruiz 
Table 1. A detailed summary of Jadad scores for all investigations included in our systematic review regarding the treatment of botulinum toxin injections for AGA

\begin{tabular}{|c|c|c|c|c|c|c|}
\hline \multirow[t]{2}{*}{ Study name } & \multicolumn{6}{|c|}{ Jadad criteria (yes: 1 , no: 0 ) } \\
\hline & $\begin{array}{l}\text { Was there } \\
\text { randomization } \\
\text { mentioned? }\end{array}$ & $\begin{array}{l}\text { Was double } \\
\text { blinding } \\
\text { mentioned? }\end{array}$ & $\begin{array}{l}\text { Was the method of } \\
\text { randomization } \\
\text { described? }\end{array}$ & $\begin{array}{l}\text { Was the method of } \\
\text { double blinding } \\
\text { mentioned? }\end{array}$ & $\begin{array}{l}\text { Are the fate of all } \\
\text { patients known? }\end{array}$ & $\begin{array}{l}\text { total } \\
\text { study } \\
\text { score }\end{array}$ \\
\hline Freund et al. [18] & 0 & 0 & 0 & 0 & 0 & 0 \\
\hline Singh et al. [19] & 0 & 0 & 0 & 0 & 1 & 1 \\
\hline Zhang et al. [20] & 0 & 0 & 0 & 0 & 1 & 1 \\
\hline Shon et al. [21] & 0 & 0 & 0 & 0 & 0 & 0 \\
\hline Zhou et al. [22] & 1 & 0 & 1 & 0 & 1 & 3 \\
\hline
\end{tabular}

AGA, androgenic alopecia.

\section{Inclusion and Exclusion Criteria}

Following the PRISMA guidelines (online suppl. Table S1; see www.karger.com/doi/10.1159/000518574 for all online suppl. material), both authors independently screened all search results for relevant abstracts and titles. Full texts were reviewed to determine inclusion eligibility. Articles were included if they (a) featured AGA subjects, and (b) performed intradermal or intramuscular injections of botulinum toxin into the scalp as an intervention for AGA. Articles were excluded if they featured neither original data nor AGA-affected subjects.

Quality of Evidence

Study quality was assessed using Jadad scoring - also known as the Oxford scale.

\section{Results}

Five clinical studies matched our criteria for inclusion (Fig. 1). Four studies were prospective cohorts while 1 was a randomized clinical trial. Four studies did not disclose any financial support from governmental or commercial entities, while one study received a grant for funding. Jadad scores ranged from 0 to 3 , with a mean of 1 (Table 1) [18-22].

In total, there were 165 subjects - all of whom were males with AGA between the ages of 19 and 57. Four studies included subjects presenting with a NorwoodHamilton gradient II to IV, whereas 1 study enrolled subjects according to the basic and specific classification [1822].

Study durations ranged from 24 to 60 weeks. The number of botulinum toxin injection sessions ranged from 1 to 5 and included 30-150 units per session, with the frequency of injections spanning 3 weeks to 5 months. One study investigated intradermal injections directly into balding regions, whereas 4 studies investigated intramuscular injections into the frontalis, temporalis, periauricular, and occipitalis [18-22].

Three studies measured hair count changes over a fixed area, while 1 study measured percent changes to hair counts but did not specify a method of data collection. Another study used graded photographic assessment as the primary end point. Of the 4 studies that measured response rates (i.e., the percentage of subjects with $>0 \%$ hair count changes), response rates ranged from $75 \%$ to $79.1 \%$. Within studies measuring hair counts before and after intramuscular injections, percent hair count changes ranged from 18 to $20.9 \%$. When administered alongside oral finasteride, response rates and hair count changes were 84.8 and $31.3 \%$, respectively. In the singular study examining hair count changes following intradermal injections, percent hair count changes were $5.1 \%$ [18-22]. Each study is described below and summarized in Table 2.

\section{Investigations}

A 2010 pilot study conducted by Freund et al. [18] investigated the effects of intramuscular botulinum toxin type A injections on men with AGA. Fifty subjects aged 19-57 were recruited in Canada with AGA classifications of Norwood II-IV and with no history of hair loss treatments in the last year. Subjects received 150 units of botulinum toxin type A injected intramuscularly into the frontalis, temporalis, periauricular, and occipitalis muscles in 2 total sessions spread 5 months apart. Doses were divided equally over 30 injection sites. Forty subjects completed the study. From baseline to study completion (i.e., 48 weeks), hair density within a $2-\mathrm{cm}$ fixed area increased by an average of $18 \%(p<0.0001)$. The overall 


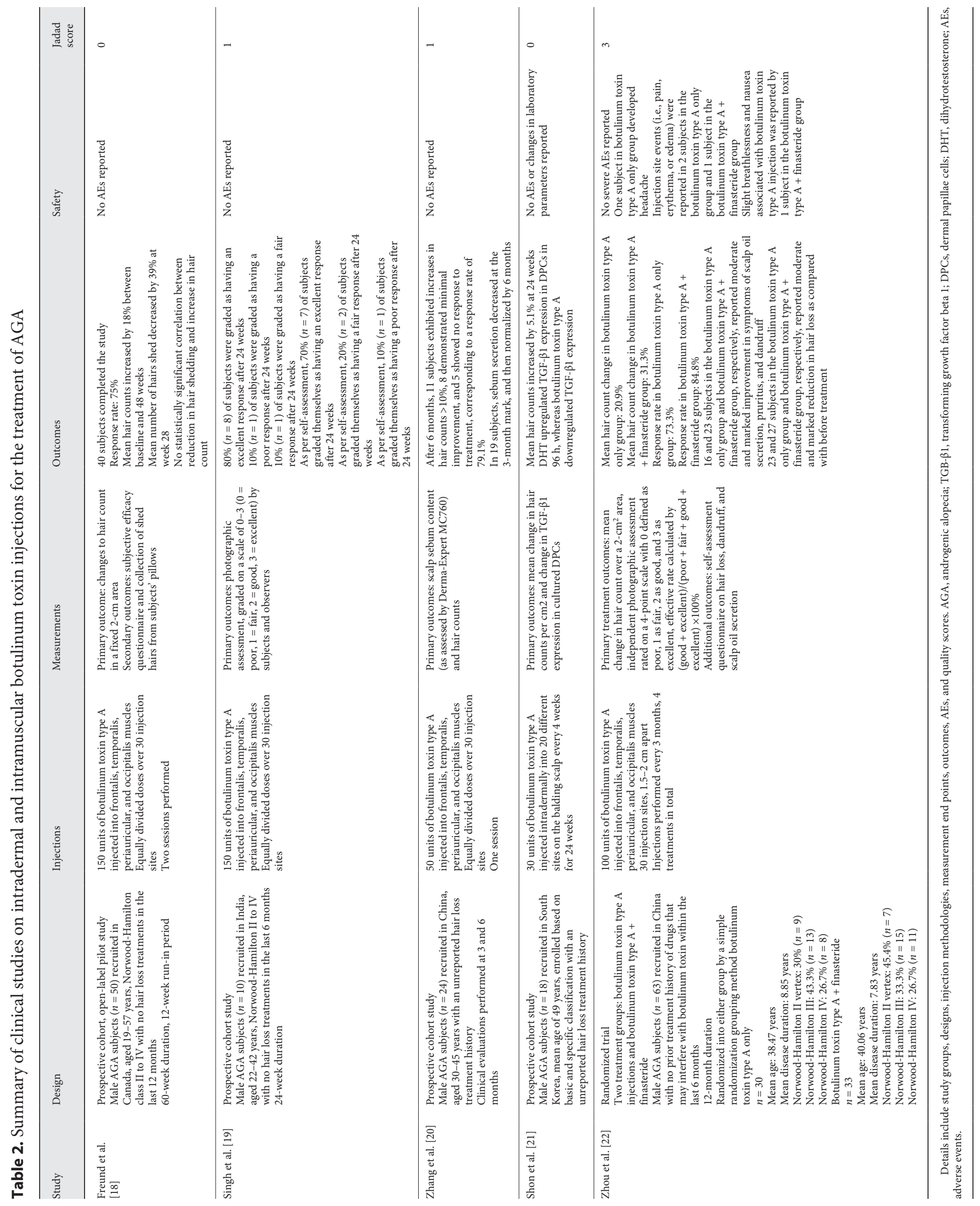


response rate, defined by investigators as participants who achieved hair count changes $>0 \%$, was determined to be $75 \%$. No adverse events (AEs) were reported.

Singh et al. [19] performed injections of 150 units botulinum toxin type A into the frontalis, temporalis, periauricular, and occipitalis muscles, equally divided over 30 injections sites, in men with AGA. Ten subjects aged 2242 years were recruited in India with AGA classifications of Norwood II-IV, and with no history of hair loss treatments in the last 6 months. The study involved a singular injection session with measurement end points determined at 24 weeks. Subject response was assessed by both photographic assessment and subject self-assessment. According to photographic assessment, 8 (80\%) subjects were determined as displaying an excellent response to the treatment, $1(10 \%)$ subject as fair, and $1(10 \%)$ subject as poor. As per self-assessment, 7 (70\%) subjects described themselves as displaying a good-to-excellent response, $2(20 \%)$ subjects graded themselves as having a fair response, and $1(10 \%)$ subject graded his response as poor. No AEs were reported.

In a prospective cohort study, Zhang et al. [20] assessed the effects of a smaller dose of botulinum toxin type A (i.e., 50 units) in 25 AGA-affected Chinese men, aged 30-45 years, with an unreported hair loss treatment history. The dose was equally divided over a minimum of 30 injection sites in 1 singular session. At a 6-month assessment, 11 (44\%) subjects exhibited hair count changes $>10 \%, 8$ (32\%) demonstrated minimal improvement, and $5(20 \%)$ showed no response to treatment - corresponding to a response rate of $79.1 \%$. No AEs were reported.

Likewise, Shon et al. [21] assessed the effects of botulinum toxin type A in AGA-affected men in South Korea. Eighteen males were recruited with a mean age of 49 years and an unreported hair loss treatment history. Subjects received 30 units of botulinum toxin type A injected intradermally and divided over 20 injection sites. Sessions were performed every 4 weeks for 24 weeks. By 24 weeks, hair per square centimeter increased significantly $(p=$ 0.012 ) from 129.61 to 136.22 , corresponding to a mean percent change of $5.1 \%$. Shon et al. [21] also performed an investigation into the effects of botulinum toxin type A on TGF- $\beta 1$ in human DPCs. Botulinum toxin type A was found to downregulate the expression of TGF- $\beta 1$, whereas DHT was found to upregulate its expression. No AEs were reported.

Finally, Zhou et al. [22] performed a randomized trial to assess botulinum toxin type A injections as a monotherapy and in combination with oral finasteride for efficacy and safety in AGA. In total, 63 men aged 18-60 years were recruited in China with AGA classifications of Norwood II-IV, and with no prior treatment history of drugs that may interfere with botulinum toxin in the last 6 months. Subjects were then randomized to receive either botulinum toxin type A injections alone $(n=30)$ or in conjunction with $1 \mathrm{mg}$ of oral finasteride daily $(n=33)$. Both groups received intramuscular injections 100 units of botulinum toxin type A into the frontalis, temporalis, periauricular, and occipitalis muscles, divided over 30 injection sites. A total of 4 sessions were performed every 3 months, for total study duration of 12 months. In the botulinum toxin type A only group, hair counts increased significantly $(p<0.001)$ from $180.57 \pm 26.53$ to $218.26 \pm$ 30.59 , for a mean percent change of $20.9 \%$. In the combination botulinum toxin type $\mathrm{A}$ and finasteride group, hair counts increased significantly $(p<0.001)$ from $178.21 \pm$ 24.33 to $234.01 \pm 27.35$, for a mean percent change of $31.3 \%$. After receiving botulinum toxin, 5 subjects reported transient side effects (i.e., headaches, injection site pain and/or swelling, and nausea). No severe AEs were reported.

\section{Discussion}

All 5 studies assessing the use of botulinum toxin in male AGA subjects demonstrated favorable outcomes, and across a range of Hamilton-Norwood gradients, injection methodologies, and study durations (Table 2). However, due to the absence of control groups, small numbers of participants, and relatively low mean Jadad scores, caution must be exercised in interpreting these results. Here, we discuss the proposed mechanisms behind botulinum toxin for AGA, limitations in the current body of research and design considerations for future studies.

\section{Mechanisms}

The mechanisms by which botulinum toxin may improve AGA are not yet established. However, there is speculation of at least 2 actions: (a) decreased TGF- $\beta 1$ activity in DPCs (through intradermal injections), and (b) relaxation of the scalp perimeter muscles (through intramuscular injections).

Research implicates the secretion of androgen-induced TGF- $\beta 1$ in the progression of AGA - particularly in regard to DPC-related hair growth inhibition, anagen phase shortening, and hair follicle miniaturization [2-4]. Interestingly, Shon et al. [21] found that botulinum toxin type A downregulated TGF- $\beta 1$ expression in cultured human DPCs - suggesting that decreased TGF- $\beta 1$ activity 
may partly explain the $5.1 \%$ increase in hair counts from intradermal botulinum toxin injections in their 24-week study. Since hair diameter was not a measured end point, it is unclear whether intradermal injections also affected hair follicle miniaturization.

While intramuscular injections of botulinum toxin have demonstrated $18-20.9 \%$ increases in hair counts, no mechanistic data exist to explain these improvements. As such, researchers have hypothesized 2 possibilities: (a) the unpinching of arterial branches that indirectly support balding regions and (b) the reduction of tension across the galea aponeurotica.

First, it is established that balding scalps exhibit lower blood flow versus controls [11]. However, reduced blood flow is often regarded as a consequence of AGA rather than a cause. This is because DPC microcapillary networks appear to collapse in late catagen - and only after a hair disconnects from its DPC cluster [23]. Moreover, AGA-related hair follicle miniaturization appears to be a single-step process: it occurs during re-entry into anagen, whereby fewer DPCs populate to produce a smaller hair follicle, thus requiring less microvascular support [24]. However, Goldman et al. [9] found that transcutaneous oxygen levels in frontal balding scalps were $54 \%$ than that of controls, and then proposed an additional explanation for microvascular insufficiency: scalp anatomy. In particular, muscular contractions of the frontalis, occipitalis, and temporalis might pinch vascular networks stemming from the supraorbital, supratrochlear, and carotid arterial branches, thus leading to a hypoxic state in baldingprone regions where DHT conversion becomes favored over estradiol. This hypothesis was reintroduced by Freund et al. [18] to explain the hair count increases from their pilot study on intramuscular botulinum injections. Interestingly, Kato et al. [25] recently demonstrated that local ischemia reduces hair shaft diameter, delays hair shaft elongation, decreases hair density, and terminates anagen. If these local ischemic effects are demonstrated in vivo in AGA subjects by way of muscular contractions, this would further strengthen Goldman et al.'s [9] hypothesis.

Second, intramuscular injections may improve AGA by reducing tension across the galea aponeurotica. Anatomically, the scalp perimeter muscles are anchored to the galea aponeurotica - a dense, fibrous, fascia-like membrane that stretches across the scalp and underlies AGA-prone regions. Two-dimensional von Mises models suggest that when the occipitalis and frontalis contract, they generate a tensile pattern across the galea that closely resembles the patterning and progression of male
AGA [26]. Tissues under pressure and/or strain induce intracellular oxidation in a dose-dependent manner, as well as increased DHT and TGF-B1 activity [15, 27, 28]. In vitro studies suggest that Hic-5/ARA55 - an androgen receptor co-activator - plays a role in both DPC androgen sensitivity and TGF- $\beta 1$ activation $[29,30]$. Hic-5/ARA55 accumulates in the nucleus, where it can promote the transactivation of androgen-specific genes alongside cell adhesion kinase beta - the latter of which is activated by mechanical stretch in vascular smooth muscle cells [31, 32]. Thus, tension-mediated stretch across the galea aponeurotica might induce inflammation that transactivates to upper layers of scalp tissue, potentiating increased DHT and TGF- $\beta 1$ activity in DPCs alongside the acceleration of AGA progression [33]. If true, intramuscular botulinum toxin injections into the frontalis, temporalis, occipitalis, and periauricular muscles might reduce galeal tension and, in doing so, improve AGA outcomes. However, it bears repeating that all intramuscular mechanisms remain hypothetical. More investigations are required to elucidate the actions of botulinum toxin both intradermally and intramuscularly.

\section{Limitations}

We identified several limitations in the current body of research. First, there is no meta-analysis within this systematic review due to heterogeneity across studies regarding subject demographics, treatment methodologies, and end point assessments. This emphasizes a need for more data collection before drawing conclusions regarding the effect of botulinum toxin injections on AGA outcomes. Second, no studies included control groups. Not uncommonly, clinically controlled trials for hair loss interventions have shown inclinations toward the placebo effect, particularly for self-assessments, investigator assessments, and even hair counts, thus drawing into question the utility of treatment-only trials $[5,34]$. Third, human scalp hair counts fluctuate seasonally [35]. This presents a challenge in interpreting results - particularly in investigations with durations shorter than 12 months that do not contain a control group. Fourth, age may be a confounding factor in response to intramuscular botulinum toxin type A in AGA subjects. Younger subjects often have stronger muscles, and thus, may require higher doses of botulinum toxin to achieve adequate muscle relaxation [36]. Consequently, studies that do not segment mean response or regrowth rates by age may inadvertently skew outcomes favorably or unfavorably dependent on the age distribution. Fifth, across all studies, there was mention of only 1 female subject who received intramus-
English Jr./Ruiz 
cular botulinum toxin injections - a case that occurred outside of Zhou et al. [22]'s initial study design. Therefore, there is no controlled data on the effects of botulinum toxin injections on females with pattern hair loss. Sixth, no studies included in this review compared botulinum toxin type A injection against FDA-approved AGA treatments, such as oral finasteride or topical minoxidil. This creates uncertainty as to whether botulinum toxin injections act comparably well to standard treatment protocols. And last, no studies measured hair diameter changes as an end point. Therefore, we do not know if botulinum toxin injections have an effect on AGA's most defining characteristic: hair follicle miniaturization.

\section{Recommendations}

Future investigations into botulinum toxin for AGA should consider study durations of at least 12 months, and evaluate the therapy against a placebo group (such as saline injections), an untreated group, and/or a treatment control group (such as oral finasteride). End point assessments should include changes to hair counts and hair diameters, as well as subject and investigator assessments. Results should be segmented by age to determine a relationship between botulinum toxin dose, response rate, and regrowth rate. There is also a need to investigate the effects on botulinum toxin on females with pattern hair loss.

Investigators should continue to explore intradermal versus intramuscular injections to elucidate which methods produce the best outcomes, and the mechanisms behind those outcomes. The number of botulinum toxin units, sessions, and frequencies of sessions should be explored to establish a set of best practices for providers and patients. Last, investigators should consider whether different serotypes of botulinum toxin influence results.

\section{Conclusion}

Five clinical studies assessing the effects of intramuscular and/or intradermal botulinum toxin injections for AGA subjects have demonstrated favorable outcomes. However, due to the absence of control groups, small numbers of participants, and relatively low mean Jadad scores, results should be interpreted with caution. There is lacking clarity surrounding the actions by which intradermal and intramuscular botulinum toxin injections might improve AGA. However, there is mechanistic support showing that intradermal injections downregulate TGF- $\beta 1$ in DPCs, and speculation that intramuscular in- jections unclamp pinched arterial passageways to AGAaffected regions as well as reduce scalp tension. Future investigations should work toward clarifying these mechanisms, improving clinical trial designs, trialing botulinum toxin in female pattern hair loss subjects, and establishing best practices for injection locations, units, sessions, and frequencies of sessions. Until botulinum toxin injections are clinically compared against approved treatments, they should remain an adjunct therapy rather than stand-alone therapy for those with AGA.

\section{Statement of Ethics}

The paper is exempt from Ethical Committee approval since it is a review article and does not include previously unpublished data on human subjects.

\section{Conflict of Interest Statement}

Robert English and Sophia Ruiz have no conflicts of interest to declare.

\section{Funding Sources}

The authors did not receive funding for this research.

\section{Author Contributions}

All authors made substantial contributions to conception and design, acquisition of data, or analysis and interpretation of data; took part in drafting the article or revising it critically for important intellectual content; agreed to submit to the current journal; gave final approval of the version to be published; and agree to be accountable for all aspects of the work.

\section{References}

1 Lolli F, Pallotti F, Rossi A, Fortuna MC, Caro G, Lenzi A, et al. Androgenetic alopecia: a review. Endocrine. 2017 Jul;57(1):9-17.

2 Inui S, Fukuzato Y, Nakajima T, Yoshikawa $\mathrm{K}$, Itami S. Androgen-inducible TGF-betal from balding dermal papilla cells inhibits epithelial cell growth: a clue to understand paradoxical effects of androgen on human hair growth. FASEB J. 2002 Dec;16(14):1967-9.

3 Inui S, Fukuzato Y, Nakajima T, Yoshikawa $\mathrm{K}$, Itami S. Identification of androgen-inducible TGF-betal derived from dermal papilla cells as a key mediator in androgenetic alopecia. J Investig Dermatol Symp Proc. 2003 Jun; 8(1):69-71. 
4 Winiarska A, Mandt N, Kamp H, Hossini A, Seltmann H, Zouboulis CC, et al. Effect of 5alpha-dihydrotestosterone and testosterone on apoptosis in human dermal papilla cells. Skin Pharmacol Physiol. 2006;19(6):311-21.

5 Kaufman KD, Dawber RP. Finasteride, a Type 25 alpha-reductase inhibitor, in the treatment of men with androgenetic alopecia. Expert Opin Investig Drugs. 1999 Apr;8(4):403-15.

6 Kaufman KD, Rotonda J, Shah AK, Meehan AG. Long-term treatment with finasteride 1 $\mathrm{mg}$ decreases the likelihood of developing further visible hair loss in men with androgenetic alopecia (male pattern hair loss). Eur J Dermatol. 2008 Jul-Aug;18(4):400-6.

7 Olsen EA, Whiting D, Bergfeld W, Miller J, Hordinsky M, Wanser R, et al. A multicenter, randomized, placebo-controlled, doubleblind clinical trial of a novel formulation of $5 \%$ minoxidil topical foam versus placebo in the treatment of androgenetic alopecia in men. J Am Acad Dermatol. 2007 Nov;57(5): 767-74.

8 Olsen EA, Weiner MS, Amara IA, DeLong ER. Five-year follow-up of men with androgenetic alopecia treated with topical minoxidil. J Am Acad Dermatol. 1990 Apr;22(4):643-6.

9 Goldman BE, Fisher DM, Ringler SL. Transcutaneous PO2 of the scalp in male pattern baldness: a new piece to the puzzle. Plast Reconstr Surg. 1996 May;97(6):1109-17; discussion 17.

10 Tellez-Segura R. Involvement of mechanical stress in androgenetic alopecia. Int J Trichology. 2015 Jul-Sep;7(3):95-9.

11 Klemp P, Peters K, Hansted B. Subcutaneous blood flow in early male pattern baldness. J Invest Dermatol. 1989 May;92(5):725-6.

12 Kasasa SC, Soory M. The effect of interleukin-1 (IL-1) on androgen metabolism in human gingival tissue (HGT) and periodontal ligament (PDL). J Clin Periodontol. 1996; 23(5):419-24.

13 Cockerham KP, Hidayat AA, Brown HG, Cockerham GC, Graner SR. Clinicopathologic evaluation of the Mueller muscle in thyroid-associated orbitopathy. Ophthalmic Plast Reconstr Surg. 2002 Jan;18(1):11-7.

14 Pagnotta A, Specchia N, Greco F. Androgen receptors in Dupuytren's contracture. J Orthop Res. 2002 Jan;20(1):163-8.
15 Hegarty P, Watson RW, Hegarty NJ, Coffey RN, Fitzpatrick JM. Pressure effects on cellular systems: is there a link with benign prostatic hyperplasia? Urology. 2004 Aug;64(2): 195-200.

16 Dutta SR, Passi D, Singh M, Singh P, Sharma $S$, Sharma A. Botulinum toxin the poison that heals: a brief review. Natl J Maxillofac Surg. 2016 Jan-Jun;7(1):10-6.

17 Ataran R, Bahramian A, Jamali Z, Pishahang V, Sadeghi Barzegani H, Sarbakhsh P, et al. The role of botulinum toxin $\mathrm{A}$ in treatment of temporomandibular joint disorders: a review. J Dent. 2017 Sep;18(3):157-64.

18 Freund BJ, Schwartz M. Treatment of male pattern baldness with botulinum toxin: a pilot study. Plast Reconstr Surg. 2010 Nov;126(5): 246e-8e.

19 Singh S, Neema S, Vasudevan B. A pilot study to evaluate effectiveness of botulinum toxin in treatment of androgenetic alopecia in males. J Cutan Aesthet Surg. 2017 Jul-Sep;10(3): 163-7.

20 Zhang L, Yu Q, Wang Y, Ma Y, Shi Y, Li X. A small dose of botulinum toxin A is effective for treating androgenetic alopecia in Chinese patients. Dermatol Ther. 2019 Jul;32(4): e12785.

21 Shon U, Kim MH, Lee DY, Kim SH, Park BC The effect of intradermal botulinum toxin on androgenetic alopecia and its possible mechanism. J Am Acad Dermatol. 2020 Dec;83(6): 1838-9.

22 Zhou Y, Yu S, Zhao J, Feng X, Zhang M, Zhao Z. Effectiveness and safety of botulinum toxin type $\mathrm{A}$ in the treatment of androgenetic alopecia. Biomed Res Int. 2020;2020:1501893.

23 Jahoda CA. Cellular and developmental aspects of androgenetic alopecia. Exp Dermatol. 1998 Oct;7(5):235-48.

24 Krajcik RA, Vogelman JH, Malloy VL, Orentreich N. Transplants from balding and hairy androgenetic alopecia scalp regrow hair comparably well on immunodeficient mice. J Am Acad Dermatol. 2003 May;48(5):752-9.

25 Kato H, Kinoshita K, Saito N, Kanayama K, Mori M, Asahi N, et al. The effects of ischemia and hyperoxygenation on hair growth and cycle. Organogenesis. 2020 Jul 2;16(3):83-94.
26 Tellez-Segura R. Involvement of mechanical stress in androgenetic alopecia. Int J Trichology. 2015 Jul-Sep;7(3):95.

27 Wang DS, Proffit D, Tsao PS. Mechanotransduction of endothelial oxidative stress induced by cyclic strain. Endothelium. 2001; 8(4):283-91.

28 Chatterjee S, Fisher AB. Mechanotransduction: forces, sensors, and redox signaling. Antioxid Redox Signal. 2014 Feb 20;20(6):86871.

29 Inui S, Fukuzato Y, Nakajima T, Kurata S, Itami S. Androgen receptor co-activator Hic-5/ ARA55 as a molecular regulator of androgen sensitivity in dermal papilla cells of human hair follicles. J Invest Dermatol. 2007 Oct; 127(10):2302-6.

30 Inui S, Itami S. Androgen receptor transactivity is potentiated by TGF- $\beta 1$ through Smad3 but checked by its coactivator Hic-5/ARA55 in balding dermal papilla cells. J Dermatol Sci. 2011 Nov;64(2):149-51.

31 Aoto H, Sasaki H, Ishino M, Sasaki T. Nuclear translocation of cell adhesion kinase beta/ proline-rich tyrosine kinase 2. Cell Struct Funct. 2002 Feb;27(1):47-61.

32 Iwasaki H, Yoshimoto T, Sugiyama T, Hirata Y. Activation of cell adhesion kinase beta by mechanical stretch in vascular smooth muscle cells. Endocrinology. 2003 Jun;144(6):230410.

33 English RS Jr. A hypothetical pathogenesis model for androgenic alopecia: clarifying the dihydrotestosterone paradox and rate-limiting recovery factors. Med Hypotheses. 2018 Feb;111:73-81.

34 Cho YH, Lee SY, Jeong DW, Choi EJ, Kim YJ, Lee JG, et al. Effect of pumpkin seed oil on hair growth in men with androgenetic alopecia: a randomized, double-blind, placebocontrolled trial. Evid Based Complement Alternat Med. 2014;2014:549721.

35 Randall VA, Ebling FJ. Seasonal changes in human hair growth. Br J Dermatol. $1991 \mathrm{Feb}$ 124(2):146-51.

36 Dover JS, Monheit G, Greener M, Pickett A. Botulinum toxin in aesthetic medicine: myths and realities. Dermatol Surg. 2018 Feb;44(2): $249-60$. 